\title{
Study on Influence of Branch Blind Pipe on Hydraulic Transients in Loading System of Launch Site
}

\author{
Yang GAO ${ }^{1, a}$, Fu-zhou FENG ${ }^{1, b}$ and Hong-xing ZHANG $^{2, c}$ \\ ${ }^{1}$ Mechanical Engineering Department, Academy of Armored Force Engineering, Beijing, China; \\ ${ }^{2}$ Taiyuan Launching Base of Satellites, Shanxi, China \\ aalexander_gao@hotmail.com ${ }^{\mathrm{b}}$ fuzhou123@163.com ${ }^{\mathrm{c}}$ hongxingz@163.com
}

\begin{abstract}
Keywords: Loading System; Branch Blind Pipe; Hydraulic Transient Process
Abstract. Influence of branch blind pipe in propellant loading system of rocket launch site on hydraulic transient in pipes is studied using computational fluid dynamics (CFD), and effects of branch blind pipe's existence, geometrical size as well as its location on hydraulic transient is analyzed, results reveal that (1) the influence of branch blind pipe on hydraulic transient is significant, and (2) relatively short branch blind pipe would increase the transient pressure peak, but longer the branch blind pipe is, less the increasing magnitude is, and slower the pressure fluctuation damping is, (3) meanwhile smaller diameter of blind pipe would also lead to higher transient pressure peak, but faster pressure fluctuation damping, (4) additionally, the branch blind pipe's location on the main pipe has limited effect on hydraulic transient. This study is meaningful for optimizing the configuration and pipe distribution of loading system, and controlling pressure fluctuation in pipes as well as ensuring the security of loading process.
\end{abstract}

\section{Introduction}

Loading system of launch site plays a very important role in aero-vehicle's ground system, and its main duty is to transport propellant from or to vehicles as well as control propellant temperature and so on ${ }^{[1]}$. When propellant loading, different loading work needs different pipes. And loading works' transformation is fulfilled by changing valves opening condition which would undoubtedly result in hydraulic transient flow. If this process were controlled improperly, extremely high instant pressure would arise, probably causing propellant leak even pipe damages, which may lead disastrous results ${ }^{[2-5]}$.

The significant traits of loading system, comparing with other pipe networks, are its substantial pipes and the complicated way they link to each other. When propellant loading, a pipe may be cut off by a closed valve on it, but part of the cutoff pipe (PCP) may still link to circulating pipes (CP). When flow fluctuation in CP occurs due to hydraulic transient, propellant in PCP would be affected and further influence fluctuation in CP. Studies on influence factors of hydraulic transient in pipes in the past usually concentrated on actions of components which would trigger the flow fluctuation such as valves $^{[6]}$ and pumps, and dampening effect of equipment such as surge tower and check valve installed intentionally to weaken the transient pressure, little attention was paid on influence of PCP on hydraulic transient. Therefore, this paper will explore the relationship between PCP and hydraulic transient using CFD, 3-D model will be used during computing for influence details research.

\section{Fundamental Physical Model}

When propellant loading, according to the loading technique, some pipes are cut off, the others not. But the latter still links to the former, and fluid in them interacts with each other. Since then, the section of the cutoff pipe that links to the $\mathrm{CP}$ can be called branch blind pipe (BBP) according to its position and function.

In order to simplify the problem, according to the loading system's structure, the fundamental physical model can be seen in Fig. 1 , a straight pipe, $4.5 \mathrm{~m}$ in length $0.16 \mathrm{~m}$ in diameter, with a ball valve $0.5 \mathrm{~m}$ from its exit, and a short blind pipe links to it between its entrance and the ball valve. At the very beginning, the ball valve is open completely, and the pipes are filled with fluid which flow 
from the main pipe's entrance to its exit steadily. Then, the valve is closed suddenly, and the hydraulic transient is triggered.

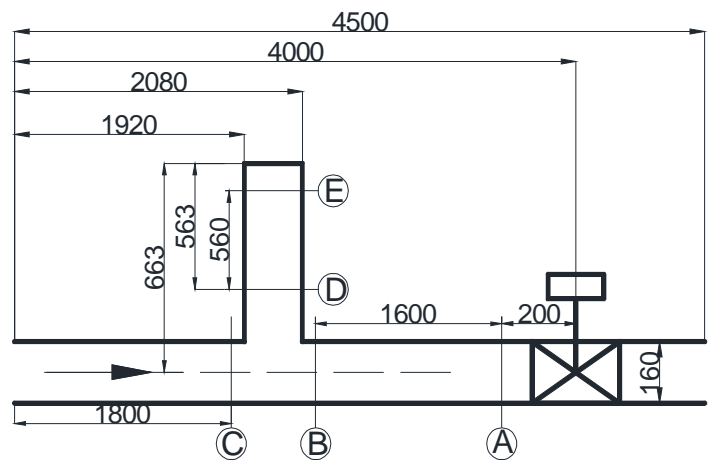

Fig.1 Fundamental physical model (unit $\mathrm{mm}$ )

\section{Computing Model}

\section{Control Equations}

Generally, only hydraulic process is considered in hydraulic transient in pipes, and fluid's compressibility should be taken into account due to local high pressure gradient. Therefore it is a 3-D compressible problem, and the control equations are

Continue equation

$$
\left.\frac{\partial \rho}{\partial t}+\nabla g \stackrel{r}{g} \rho \mathbf{u}\right)=0
$$

$\mathrm{N}-\mathrm{S}$ equation

$$
\left.\left.\frac{\partial\left(\rho u_{i}\right)}{\partial t}+\nabla g \rho u_{i}^{\mathrm{u}}\right)=-\frac{\partial p}{\partial x_{i}}+\nabla g \mu \nabla u_{i}\right) i=1,2,3
$$

State equation

$$
\left(\frac{\rho}{\rho_{0}}\right)^{n}=\frac{K}{K_{0}}
$$

Where

$$
\begin{gathered}
K=K_{0}+n \Delta p \\
\Delta p=p-p_{0}
\end{gathered}
$$

In which, $p_{0}$ is reference liquid pressure; $p$ is liquid pressure; $\rho_{0}$ is reference liquid density; $\rho$ is liquid density at pressure $p ; K_{0}$ is reference bulk modulus; $K$ is liquid bulk modulus at pressure $p ; n$ is density exponent, usually determined by experiment. Considering turbulence, with standard k-\& model, equations above make up hydraulic transient control equations.

Computation of hydraulic transient is based on the result of steady computation before the valve's closing. During computing, unsteady solver and SIMPLE algorithm are used, and second-order forward difference is chose to discretize the convection-diffusion formula. The software Fluent is used, and the time step is $10^{-4} \mathrm{~s}$, computing lasts 400 time steps. During computing, cavitation is ignored.

\section{Mesh Plot}

3-D model is established according to Fig.1, and mesh plot is implemented in computing domain using tetrahedral grid. Considering intensive changing of flow state near the valve, smaller grid is used near the valve region. Since the ball valve condition is controlled by valve shaft's rotation, the combination layer of valve passage and pipe passage will transfer to wall with valve shaft's rotation, so computing domains' link plane between valve passage and pipe passage use slide grid. Besides, grid near the wall is refined. The valve closes linearly, its rotating velocity decided in Section 2.3, and its rotating process in Fluent fulfilled by UDF program. Fig.2 shows the mesh plot result. 


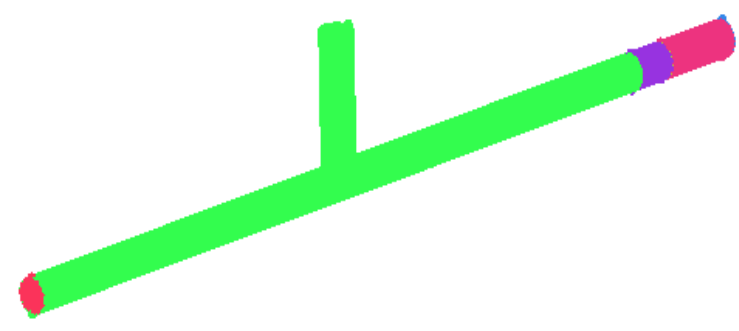

2a. Overall mesh plot result

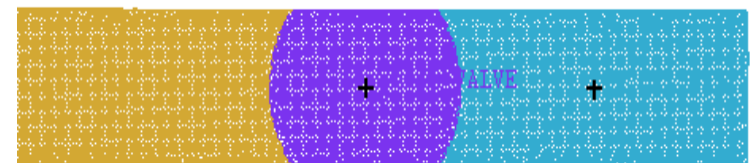

2b. Mesh near valve

\section{Boundary Condition}

Fig.2 Mesh plot result

Pressure inlet is used at the pipe entrance, in which gauge total pressure and initial gauge pressure are $5.01 \mathrm{kPa}$ and $5 \mathrm{kPa}$ respectively, with the help of above sets, the wall turbulent parameters can be received. Pressure outlet is adopted at the pipe exit, in which the gauge pressure is 0 , since the exit links to the atmosphere directly. The atmosphere pressure is set as operating pressure. And no slip shear condition is adopted for all walls.

At first, the ball valve is open completely, with the axes of valve and pipe passages coinciding with each other, suppose the angle between the axes is $\alpha$, so $\alpha=0$ under such condition, and when the valve is wholly closed, $\alpha=\alpha_{0}$. The wave velocity of hydraulic transient in pipes ${ }^{[7]}$

$$
a=\sqrt{\frac{K}{\rho[1+(K / E) \psi]}}
$$

In which $E$ is Young's modulus of elasticity of the pipe wall; $\psi$ is a nondimensional parameter that depends on the pipe's elastic properties, $\psi=0$ for rigid pipes. Taken liquid water as the computing fluid, ignoring the pipe's elasticity, the wave velocity can be estimated at about $1484.6 \mathrm{~m} / \mathrm{s}$ according liquid water's physical properties. Since the distance between valve and pipe entrance is $L=4 \mathrm{~m}$, the wave velocity's propagation period is about $2 \mathrm{~L} / \mathrm{a}=0.0054 \mathrm{~s}$. In order to simulate the hydraulic transient effectively, and avoid direct waterhammer, the valve is closed in $t=0.006 \mathrm{~s}$. Therefore the valve shaft's rotating velocity is $\alpha_{0} / t$. During computing, the valve starts closing at $0 \mathrm{~s}$, and its action is fulfilled using UDF.

\section{Result Analysis}

\section{Effect of BBP's Existence on Hydraulic Transient in Pipes}

In order to analyze the effect of BBP's existence on hydraulic transient in pipes, transient process in pipe without BBP (simple pipe, SP) and that in pipe with BBP (blind pipe, BP) are compared with each other. The BP's geometry shows in Fig.1, and SP's geometry can be received by removing the blind pipe section from BP. During computing, results at position A-E in Fig.1 will be monitored for $\mathrm{BP}$, and those at position A, B, C for SP. All data used to analyze are averages on monitor plane at specified position.

Firstly, gauge pressure at position A (before valve) is analyzed for the highest pressure is commonly arisen at the trigger's position, the position before the valve in this paper. The gauge pressure fluctuation at position A shows in Fig.3, dotted line for SP, solid line for BP. It reveals in Fig.3 that the sudden close of valve excites intense pressure fluctuation before the valve, and relatively high negative pressure appears due to expansion wave for the ignorance of cavitation. The existence of BBP intensifies both the positive and negative pressure, and slows down the fluctuation's weakening. 


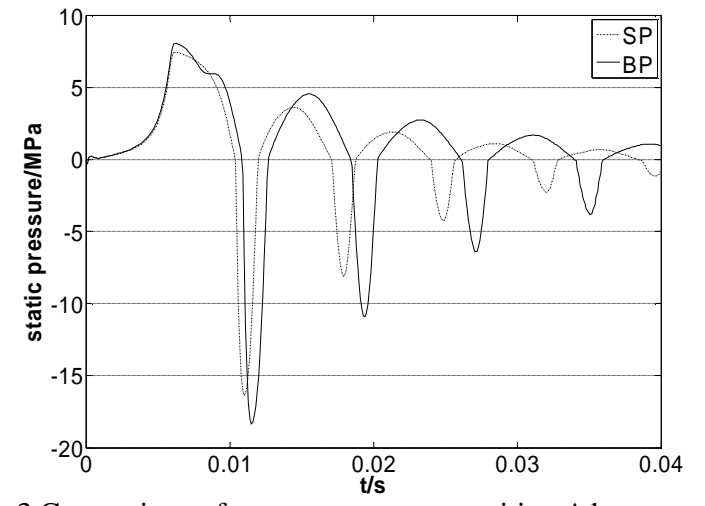

Fig. 3 Comparison of gauge pressure at position A between SP and BP

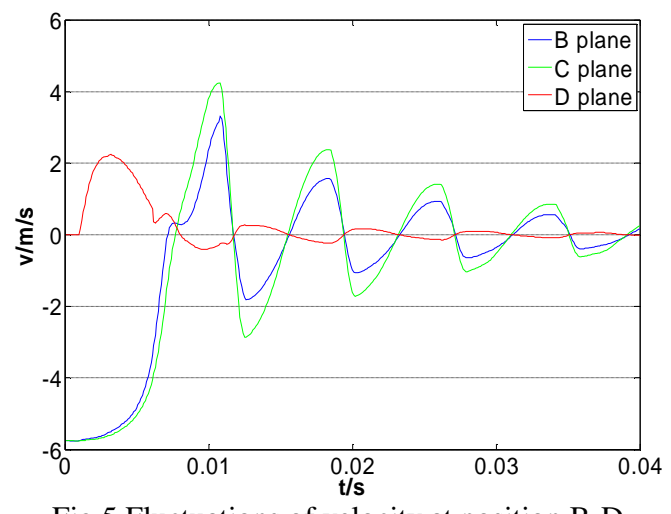

Fig.5 Fluctuations of velocity at position B-D

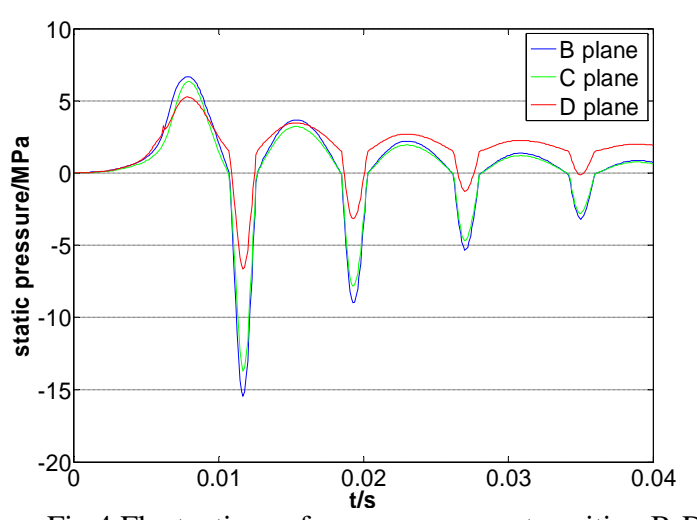

Fig.4 Fluctuations of gauge pressure at position B-D

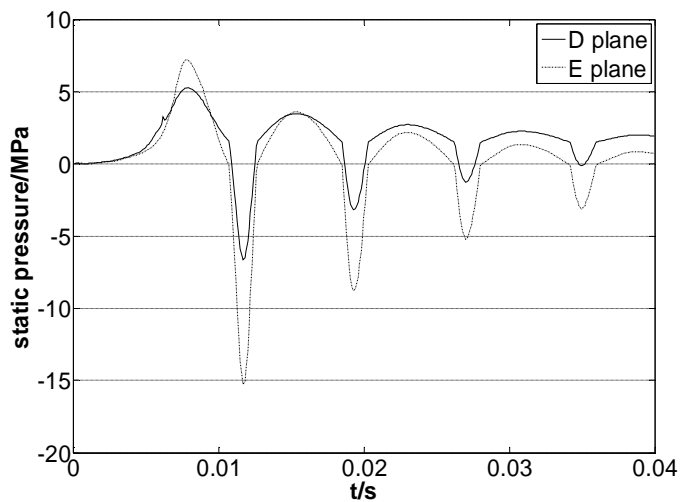

Fig.6 Comparison of fluctuation of gauge pressure at inlet and endpoint of the blind pipe

The Gauge pressure fluctuations at position B-D shows in Fig.4. There are discrepancies between pressures at position $\mathrm{D}$, the blind pipe's entrance, and at position B, C, positions after and before the link point of main pipe and blind pipe. Obviously, the upstream pressure inherits the downstream one better than the blind pipe. The average velocity fluctuations at position B-D shows in Fig.5, from which it can be seen that the upstream velocity waves more severely than the downstream one, and fluid velocity in blind pipe is tiny. Further compare gauge pressure at position $\mathrm{D}$ with that at position E, the endpoint of the blind pipe, in Fig. 6 in which dotted line is for gauge pressure fluctuation at position E. Clearly, pressure peak at position $\mathrm{E}$ is higher than that at position $\mathrm{D}$ significantly, probably due to fluid's further compression at the endpoint of the blind pipe.

\section{Effect of BBP's Length on Hydraulic Transient in Pipes}

According to section 3.1, BBP would influence the pressure peak at position A, but that how its geometrical size and location affects the transient process is still ambiguous, and the following sections will make it clear gradually. In this section, effect of BBP's length on hydraulic transient in pipes will be discussed. The gauge pressure fluctuations at position A with blind pipe length in $0.663 \mathrm{~m}, 0.885 \mathrm{~m}, 1.326 \mathrm{~m}$ and $2.200 \mathrm{~m}$ respectively show in Fig.7. Clearly, longer the blind pipe is, slower down the pressure weakening process is. And there is a subordinate pressure peak after the main pressure peak when BBP exists. It is believed that the subordinate pressure peak results from pressure reflection of blind pipe, since longer the blind pipe is, later and smaller the subordinate peak appears. The gauge pressure peaks at position A with different BBP's length are marked in Fig.8, from which it can be seen that relative short BBP would strengthen the gauge pressure, but with the increasing length, the pressure peak decreases gradually. Once the BBP's length is longer than the upstream part of the main pipe, the gauge pressure peak drops even below that of SP at position A. 


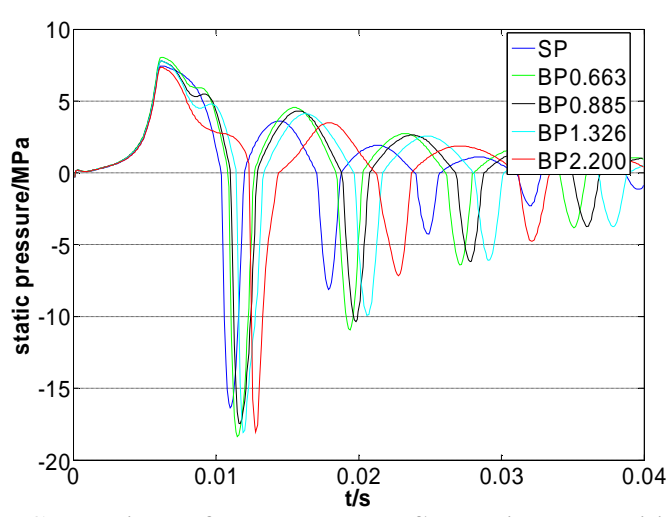

Fig.7 Comparison of gauge pressure fluctuations at position A with different BBP's length

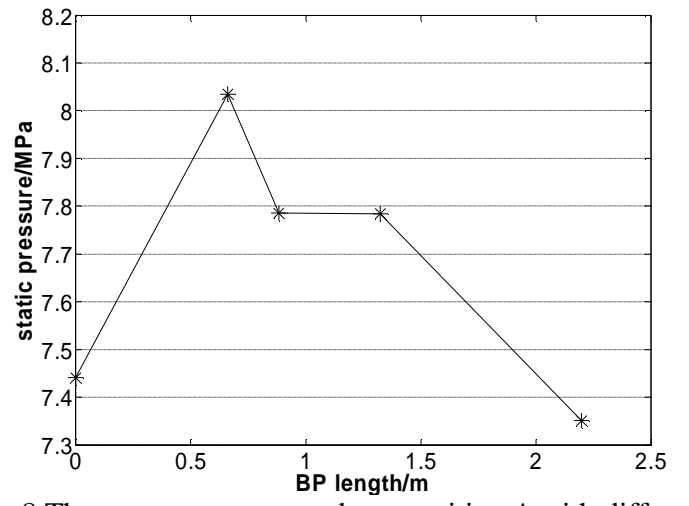

Fig.8 The gauge pressure peaks at position A with different BBP's length

\section{Effect of BBP's Diameter on hydraulic transient in pipes}

The gauge pressure fluctuations at position A with BBP's diameter in $0.16 \mathrm{~m}$ and $0.10 \mathrm{~m}$ respectively show in Fig.9, dotted line for situation of BBP's diameter in $0.16 \mathrm{~m}$. Obviously, smaller diameter will strengthen the pressure and quicken the pressure's waning.

\section{Effect of BBP's Location on hydraulic transient in pipes}

The gauge pressure fluctuations at position A with BBP's location $2 \mathrm{~m}, 3 \mathrm{~m}$ and $1 \mathrm{~m}$ from the main pipe's entrance respectively show in Fig.10. Limited effect of BBP's location on gauge pressure peak can be seen, but relatively significant effect on pressure's waning.

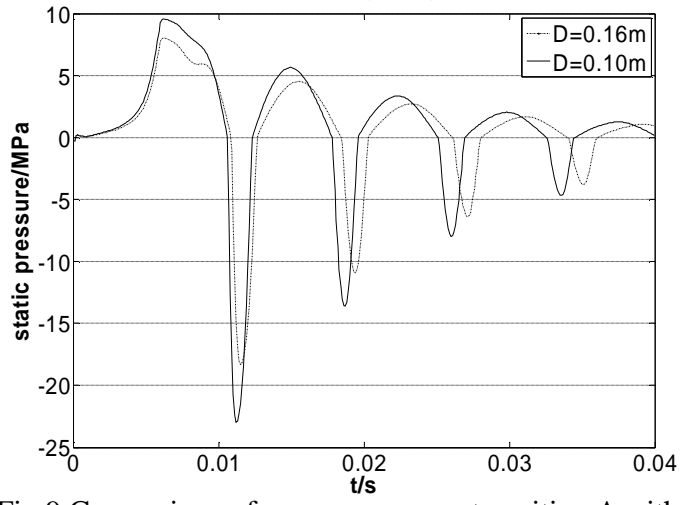

Fig. 9 Comparison of gauge pressure at position A with different BBP diameter

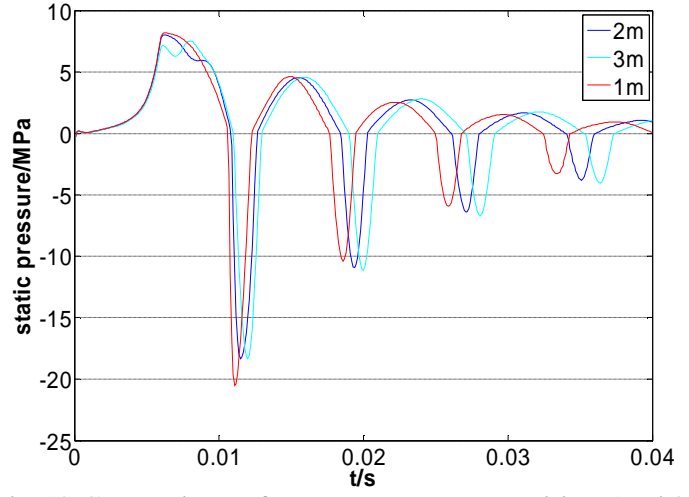

Fig.10 Comparison of gauge pressure at position A with different BBP's location

\section{Summary}

This paper studies the influence of BBP which is familiar in propellant loading system of launch site on hydraulic transient in pipes using CFD. And it analyzes how the BBP's existence, geometrical size and location affect the transient process, and the results reveal that, (1) the influence of BBP on hydraulic transient is significant, and (2) relatively short BBP would increase the transient pressure peak, but longer the branch blind pipe is, less the increasing magnitude is, and slower the pressure fluctuation damping is, (3) meanwhile smaller diameter of BBP would also lead to higher transient pressure peak, but faster pressure fluctuation damping, (4) additionally, the BBP's location on the main pipe has limited effect on hydraulic transient. Results above are meaningful for further studying transient process in pipe networks, optimizing the loading system's configuration and structure design, and controlling pressure fluctuation in pipes as well as ensuring the security of loading process.

\section{References}

[1] Zaixue ZHOU: Launch technology (China astronautic publishing house, Beijing 2009).

[2] A. Bergant, A. R. Simpson and A. S. Tijsseling: Water hammer with column separation: A historical review. Journal of fluids and structures, Vol. 22(2006), p.135-171. 
[3] A. Riasi, A. Nourbakhsh and M. Raisee: Energy dissipation in unsteady turbulent pipe flows caused by water hammer. Computers \& Fluids, Vol.73 (2013), p.124-133.

[4] Jiasheng HE: Water hammer and its prevention strategy in cryogenic system. Aerospace launch technology, Vol.90 (2007), p.34-37.

[5] W. I. Moor, R. I. Arnold: Failure of Apollo Saturn V liquid oxygen loading systems. Advances in Cryogenic Engineering, Vol.13 (1968), p.13-16.

[6] P. H. Azoury, M. Baasiri: Effect of valve-closure schedule on water hammer. Journal of Hydraulic Engineering, Vol. 112 (1986), p.890-903.

[7] Chaudhry M H: Applied hydraulic transients (Springer, New York 2014). 